\title{
Prognostic and Predictive Impact of Ki-67 in Premalignant and Malignant Squamous Cell Lesions of Oral Cavity
}

\author{
Veena Maheshwari, SC Sharma, Varsha Narula, Sachin Verma, Anshu Jain, Kiran Alam
}

\begin{abstract}
Introduction: Oral squamous cell carcinoma, the fifth most common cancer worldwide, is a major cause of morbidity and mortality in India. It most commonly occurs in middle-aged and older individuals. Typically, they tend to be preceded by a premalignant state for a long time. The present study evaluates the clinicopathological profile of patients with oral lesions and the role of malpractices that promote carcinogenesis in the oral mucosa and the use of $\mathrm{Ki}-67$ as an important predictor of the same.
\end{abstract}

Materials and methods: The expression of $\mathrm{Ki}-67$ was studied in paraffin-embedded tissue sections of oral lesions of all the cases after taking a detailed history and examination.

Results: The study was conducted on 65 patients with 25 dysplastic and 40 malignant lesions. Males were affected more frequently than females. The most common age group affected was 31 to 60 years with mean age of 45.4 years. Anterior twothird of tongue (41\%) was the most commonly affected site. $95 \%$ of the patients had a history of addiction in form of smoking, tobacco chewing and betel nut. Out of 25 cases of dysplasia, 11 showed low expression while out of 40 cases of squamous cell carcinoma only three showed low and 29 showed high expression of $\mathrm{Ki}-67$.

Conclusion: The expression of $\mathrm{Ki}-67$ correlates well with the disease progression from dysplasia to carcinoma of the oral cavity. It is therefore a marker of malignant transformation and carcinogenesis in oral premalignant lesions and in future it may serve as a prognostic tool in the early detection of malignancy.

Keywords: Oral cancer, Dysplasia, Premalignant, Ki-67.

How to cite this article: Maheshwari V, Sharma SC, Narula V, Verma S, Jain A, Alam K. Prognostic and Predictive Impact of Ki-67 in Premalignant and Malignant Squamous Cell Lesions of Oral Cavity. Int J Head Neck Surg 2013;4(2):61-65.

\section{Source of support: Nil}

Conflict of interest: None declared

\section{INTRODUCTION}

Oral cavity cancer accounts for approximately $3 \%$ of all malignancies and is a significant worldwide health problem. ${ }^{1}$ It is, in fact, one of the most common malignancies in Southeast Asia, accounting for up to 30 to $40 \%$ of all malignancies in India. ${ }^{2}$ Over $90 \%$ of these tumors are squamous cell carcinomas, which arise from the oral mucosal lining. These cancers are found to progress from premalignant/precancerous lesions, beginning as hyperplastic tissue and developing into invasive squamous cell carcinoma. The etiology of this transition is multifactorial and the important risk factors include personal habits of tobacco, smoking, alcohol and betel nut chewing. ${ }^{3}$ The area of research in the present study has a markedly high prevalence of oral cancer attributable mainly to the aforementioned risk factors. The purpose of this article is to review the clinical features of oral cancer and dysplastic lesions, with an emphasis on various risk factors that promote the process of carcinogenesis. Also discussed is the use of Ki-67 as an important marker of proliferation and hence early detection aiding quick intervention.

\section{MATERIALS AND METHODS}

The present study was carried out on 65 patients of dysplastic and malignant lesions of oral cavity admitted or attending IPD/OPD of Department of Otorhinolaryngology (ENT), JNMCH, Aligarh, Uttar Pradesh. A concise history, clinical examination, including local examination of oral region was carried out along with relevant investigations.

Histopathology of paraffin-embedded section of specimens was done using hematoxylin and eosin (H\&E) stain. Histologically proven oral dysplastic and malignant lesions were selected for the study.

Expression of Ki-67 was studied on these sections and it was correlated with the clinical staging and histological grading of the lesion. Only strong brown nuclear staining of epithelial cell was considered positive. Those histological sections with uniform and good intensity staining were assessed for scoring. The section stained for Ki-67 proliferation were evaluated using scores from 1 to 3 .

1. +++- High proliferation $\quad->50 \%$ positive cells

2. ++- Moderate proliferation -30 to $50 \%$ positive cells

3. + - Low proliferation - 10 to $30 \%$ positive cells.

\section{RESULTS}

The study of 65 selected cases indicated a predominance of neoplasia in 40/65 cases in patients aged between 61 and 70 years with a striking male predominance $(83 \%)$.

Regarding their topography, we observed that most of the cases presented with carcinoma of the anterior two-third of tongue (41.54\%) followed by floor of mouth (20\%) and other sites.

The clinical profile varied from maximum number of cases presenting with growth in the oral cavity $(38.46 \%)$ followed by white patch on the mucosa $(23.07 \%)$ and ulceration $(15.39 \%)$. 
In terms of histopathological behavior, the tumors corresponded with well-differentiated forms in 20 cases $(30.76 \%)$, moderately differentiated in 15 cases $(23.07 \%)$ and poorly differentiated in five cases $(7.69 \%)$. Twenty-five cases of dysplasia were also identified with 10 each mild and moderate dysplasia (15.38\%) and five showing severe dysplasia (7.69\%) (Table 2).

Regarding the risk factors, $62 \%$ cases in the present study had a history of addiction in the form of smoking, tobacco chewing, pan masala and betel nut chewing (Table 1).

The immunoreaction analysis of Ki-67 indicated high proliferation in 29 cases $(44.61 \%)$, moderate in eight $(12.3 \%)$ and low in three cases $(4.61 \%)$ of squamous cell carcinoma (Figs 2A to D) while moderate proliferation was seen in $14(21.53 \%)$ and low in 11 cases $(16.92 \%)$ of dysplasia (Figs 1A to D) (Table 2). The mean Ki came out to be $25.45 \pm 3.31$ for dysplasias and $45.55 \pm 6.59$ for carcinomas, the difference being statistically significant $(\mathrm{p}<0.05)$ (Table 3). Only strong brown nuclear staining of epithelial cell was considered positive.

\section{DISCUSSION}

The incidence of oral cancer remains high and is associated with many deaths in both Western and Asian countries. More than 300,000 new cases worldwide are being diagnosed with oral squamous cell carcinoma annually. Oral cancer is estimated by WHO to be the eighth most common cancer worldwide. In India and other Asian countries, oral and oropharyngeal carcinomas comprise up to half of all malignancies, with this particularly high prevalence being attributed to the influence of carcinogens and region-specific epidemiological factors, especially tobacco and betel quid chewing., ${ }^{4,5}$

In our study, $95 \%$ of the patients were under the influence of potential risk factors like tobacco, alcohol and betel nut. Subapriya et al in their study in 2007 also established a causal association between these factors and oral cancer. ${ }^{6}$ Similar results were obtained by Warnakulasriya et al in $2005 .{ }^{7}$ All these factors bring about structural changes in DNA of cells which if go unnoticed lead to cancer.

Dietary deficiency of vitamin A, vitamin C, vitamin E, iron, selenium, folate and other trace elements have been linked to increased risk of oral cancer. Poor oral hygiene and ill-fitting dentures may also play a role in localizing a site where tumors may develop. However, both of these factors are unlikely to cause oral cancer in the absence of other risk factors. ${ }^{8}$

The presence of epithelial dysplasia is generally accepted as one of the most important predictors of malignant development in premalignant lesions. This was originally based on follow-up studies on cervical lesions done by Richart and Barron (1969), and later on findings that oral lesions with epithelial dysplasia more often develop into carcinoma than those without dysplasia as reported by Pindborg et al (1997), Burkhardt and Maerker (1978), Kramer et al (1978) and others. However, epithelial dysplasias will not necessarily develop into cancer, and some may even regress as documented in various studies done by Mincer et al (1972), Silverman et al (1976), Bánóczy and Csiba (1976) and Gupta et al(1980). Burkhardt and Maerker (1978) reported that mild, moderate, and severe dysplasias develop into malignancy in 3,4 , and $43 \%$ respectively. ${ }^{9}$ The present study documented 10 cases each of mild and moderate dysplasia while five of poor dysplasia (Table 2).

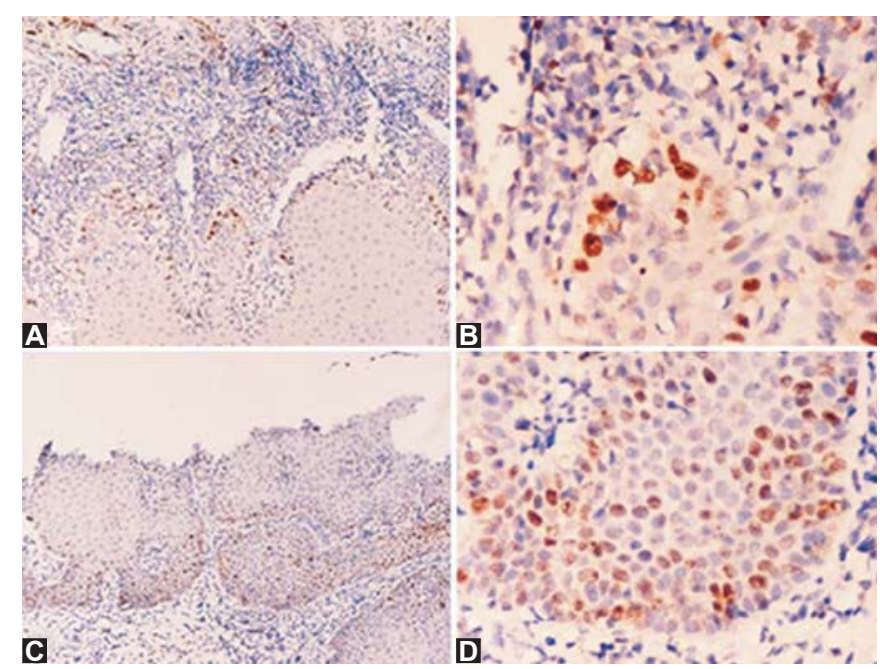

Figs 1A to D: (A) Leukoplakia showing brown nuclear staining confined to only basal layer Ki-67, IHC (x100), (B) leukoplakia showing brown nuclear staining confined to only basal layer Ki-67, IHC (x400), (C) moderate dysplasia showing scattered brown nuclear stain. Ki-67, IHC $\times 100$, (D) moderate dysplasia showing scattered brown nuclear stain. $\mathrm{Ki}-67, \mathrm{IHC} \times 400$

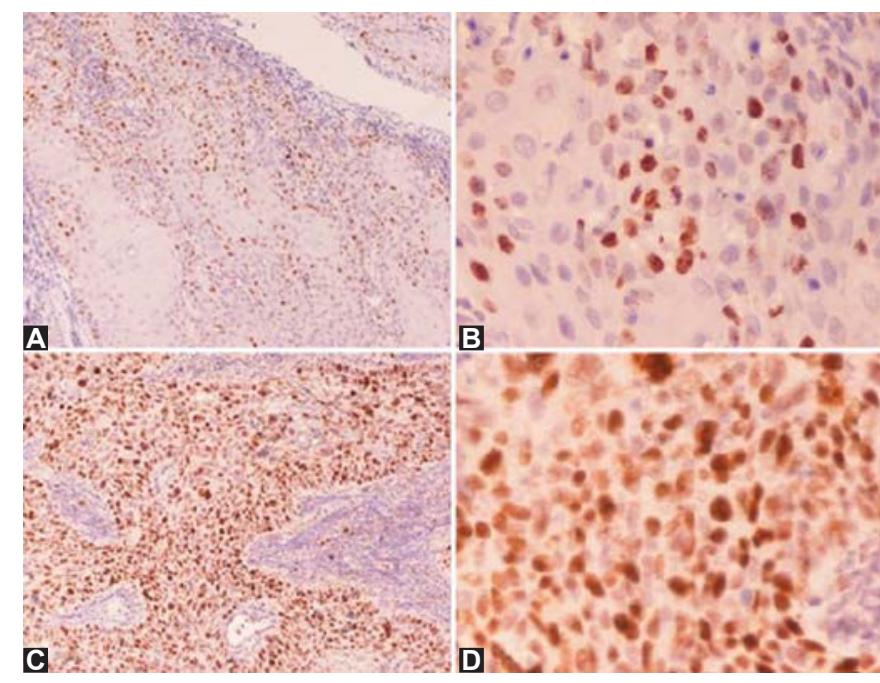

Figs 2A to D: (A) Well-differentiated squamous cell carcinoma [showing scattered Ki-67 expression. IHC $(\times 100)$, high proliferation], (B) well-differentiated squamous cell carcinoma [showing scattered Ki-67 expression. IHC ( $\times 400)$, high proliferation], (C) poorly differentiated squamous cell carcinoma showing dense nuclear staining Ki-67, IHC $\times 100$, (D) poorly differentiated squamous cell carcinoma showing dense nuclear staining $\mathrm{Ki}-67, \mathrm{IHC} \times 400$ 
In our study, majority of patients, 25 cases (38.46\%) presented with symptom of growth in oral cavity followed by 15 cases $(23.07 \%)$ of white/red patches in oral mucosa. Symptoms vary according to the site of the tumor. White or red patches on the oral mucosa may indicate a precancerous condition and a biopsy is essential. Up to $6 \%$ of leukoplakias may become malignant over a 10 -year period. ${ }^{10}$

A recent Cochrane review concluded that there is no effective treatment, preventing malignant transformation of leukoplakia. Treatment using chemoprevention with vitamin $\mathrm{A} /$ retinoid analogous is associated with high rate of clinical resolution but there is a high rate of relapse when therapy is discontinued and the side effects of the treatment mean it is not applicable as a preventive treatment in the population groups at risk. ${ }^{11}$

Hyperproliferation is thought to be an early marker of disorderly growth. It is generally accepted that increased proliferation is associated with more advanced lesions and that the distribution of proliferating cell in tissue may reveal more about the regulatory mechanism that become dysfunctional during the multistep process of carcinogenesis. $^{12}$

The immunohistochemical markers used to study cell proliferation are PCNA, Ki-67, cyclin D and CENP-F. The present study focuses on one of the proliferating markers Ki-67, as stained by MIB-1 which is easy to interpret because there is low background staining and the nuclear stain is very intense. It is also believed to be a reliable marker of cell proliferation because this nonhistone protein can be expressed at all stages of the cell cycle except $\mathrm{G} 0 .^{13}$

$\mathrm{Ki}-67$ human nuclear antigen is expressed during the $\mathrm{G} 1$, $\mathrm{S}, \mathrm{G} 2$ and M phases of the cell cycle, but is absent in the quiescent $\mathrm{G} 0$ phase. It is localized in the nucleoli in G1, with a nucleoplasmic distribution later in the cycle, increasing in intensity through $\mathrm{S}$ and $\mathrm{G} 2$, to reach a maximum at mitosis. In the $\mathrm{S}$ phase the antigen was also detected homogenously in the karyoplasms and in the G2 phase, the staining within the karyoplasms existed as a mixed, finely granular, and speckled pattern with perinucleolar staining still present. Intense perichromosamal Ki-67 staining was noted during

\begin{tabular}{lllllll}
\multicolumn{7}{c}{ Table 1: Distribution according to oral habits $(\mathrm{n}=65)$} \\
\hline Addictions & Mild dysplasia & $\begin{array}{l}\text { Moderate } \\
\text { dysplasia }\end{array}$ & $\begin{array}{l}\text { Severe } \\
\text { dysplasia }\end{array}$ & $\begin{array}{l}\text { Well-differentiated } \\
\text { squamous cell } \\
\text { carcinoma }\end{array}$ & $\begin{array}{l}\text { Moderately } \\
\text { differentiated }\end{array}$ & $\begin{array}{l}\text { Poorly differentiated } \\
\text { squamous cell } \\
\text { carcinoma }\end{array}$ \\
\hline No addiction & $1(1.54 \%)$ & - & - & - & $2(3.07 \%)$ & - \\
Smoking & $1(1.54 \%)$ & $2(3.07 \%)$ & - & $3(4.61 \%)$ & $3(4.61 \%)$ & $2(3.07 \%)$ \\
Tobacco chewing & $2(3.07 \%)$ & $2(3.07 \%)$ & $1(1.54 \%)$ & $4(6.15 \%)$ & $1(1.54 \%)$ & - \\
Alcohol & - & - & $1(1.54 \%)$ & $1(1.54 \%)$ & $2(3.07 \%)$ & - \\
Pan masala (Gutkha) & $1(1.54 \%)$ & $2(3.07 \%)$ & $2(3.07 \%)$ & $5(7.69 \%)$ & $1(1.54 \%)$ & $1(1.54 \%)$ \\
Pan, betel nut chewing & $3(4.61 \%)$ & $2(3.07 \%)$ & - & $5(7.69 \%)$ & $2(3.07 \%)$ & $1(1.54 \%)$ \\
Combination of either & $2(3.07 \%)$ & $2(3.07 \%)$ & $1(1.54 \%)$ & $2(3.07 \%)$ & $4(6.15 \%)$ & $1(1.54 \%)$ \\
\hline Total & $10(15.38 \%)$ & $10(15.38 \%)$ & $5(7.69 \%)$ & $20(30.76 \%)$ & $15(23.07 \%)$ & $5(7.69 \%)$ \\
\hline
\end{tabular}

Table 2: Ki-67 expression in dysplasias and carcinomas

\begin{tabular}{lcccc}
\hline Category & Cases & Low & Moderate & High \\
\hline Mild dysplasia & 10 & 8 & 2 & - \\
Moderate dysplasia & 10 & 2 & 8 & - \\
Severe dysplasia & 5 & 1 & 4 & - \\
Well-differentiated squamous cell carcinoma & 20 & 3 & 3 & 14 \\
Moderately differentiated squamous cell carcinoma & 15 & - & 5 & 10 \\
Poorly differentiated squamous cell carcinoma & 5 & - & - & 5 \\
\hline
\end{tabular}

Table 3: Mean Ki-67 labeling index scores

\begin{tabular}{llcc}
\hline & Groups & Cases & Mean Ki-67 labeling index scores \\
\hline \multirow{2}{*}{ Dysplasia: } & & 25 & $25.45 \pm 3.31$ \\
& Mild dysplasia & 10 & $18.56 \pm 3.01$ \\
& Moderate dysplasia & 10 & $22.23 \pm 3.55$ \\
& Severe dysplasia & 5 & $28.22 \pm 3.95$ \\
Carcinoma: & & 40 & $45.55 \pm 6.59$ \\
& & 20 & $48.52 \pm 4.63$ \\
& Well-differentiated squamous cell carcinoma & $52.23 \pm 5.52$ \\
& Moderately differentiated squamous cell carcinoma & 15 & $58.55 \pm 6.23$ \\
\hline
\end{tabular}


prophase and metaphase, in addition to karyoplasmic staining in prophase and cytoplasmic staining in metaphase. The intensity of staining then rapidly diminished during anaphase and telophase. The half life of detectable Ki-67 antigen is very short, an hour or less due to the presence of numerous proline-glutamic acid-serine-threonine (PEST) motifs in the protein that facilitate rapid catabolism. ${ }^{14,15}$

In healthy epithelium, it remained confined to isolated cells/occasional cells adjacent to the basal lamina, i.e. in the basal layer and mostly in the parabasal layer with no positivity in the superficial layer. The present study showed proliferative cells at abnormal sites within the epithelium, sometimes throughout, the entire epithelium thickness (Figs 1A to D). These findings supported those of Liu et al (1998), who found that the basal and superficial layers showed the clearest differences between normal and abnormal tissues. ${ }^{12}$

Zidar et al (1996) showed that in abnormal and atypical dysplasia, these is a significant increase in the number of Ki-67 positive cells that appeared in the suprabasal portion of the epithelium, characterized by augmentation of immature basal like cells that occupy the lower two-thirds and may extend up to the surface of the epithelium. ${ }^{16}$ Similar findings were seen in the present study (Figs 1A to C).

A report by Macluskey et al (1999) found that the correlation with the mean Ki-67 labeling index was stronger for the healthy tissue and dysplasia than for carcinomas but was significant in all three cases. The results suggest that epithelial proliferation may continue to increase during the transition from dysplasia to carcinoma, but this is likely to occur at a slow rate. ${ }^{17}$

One more reason for the increase in proliferating cells with Ki-67 has been explained by Slootweg et al (1994), who found that tumor cells are in some way able to bypass the suppressive action of the $\mathrm{p} 53$ gene. It is concluded that the appearance of $\mathrm{p} 53$ protein occurs early in carcinogenesis and that cells may also show increased proliferation without involving immunohistochemically detectable alterations in the p53 gene. ${ }^{18}$

It was found that there is a decrease in cells undergoing apoptosis in less-differentiated tumors with an increase in the number of tumor cells and of tumor growth. This decrease in apoptosis was correlated with an increase in the proliferative activity by Todd et al (2002). ${ }^{19}$

The present study showed an inverse correlation between the degree of tumor differentiation and the rate of cell proliferation obtained by the expression of Ki-67. Results similar to those found in this study are also suggested by Carreras et al (2005) in cervical carcinoma, ${ }^{20}$ Stanton et al (2003) in malignant pancreatic tumors, ${ }^{21}$ Sym et al (2011) in gastric cancers ${ }^{22}$ and Marqulis et al (2006) in transitional cell carcinoma of bladder. ${ }^{23}$
$\mathrm{Ki}-67$ is a cancer antigen that is found in growing, dividing cells but is absent in the resting phase of cell growth making it a good tumor marker. Chemotherapy drugs target cells that are growing beyond the normal rate and so these drugs can be effective on aggressive tumors.

Higher levels of Ki-67 has a good response to chemotherapy. A high index of Ki-67 usually means a poor prognosis although if the cancer responds well to appropriate chemotherapy, long-term survival is achievable.

To conclude, the expression of Ki- 67 correlates well with the clinical stage and histological grade of oral cavity carcinoma and it is a good proliferation marker in indicating aggressiveness of the tumor and it can possibly be used as a prognostic marker in chemotherapy.

It may be used either as a static proliferation marker or by making multiple measurements during treatment as a dynamic marker of treatment efficacy.

\section{REFERENCES}

1. Silverman S Jr. Demographics and occurrence of oral and pharyngeal cancers. The outcomes, the trends, the challenge. J Am Dent Assoc 2001;132 (Suppl):7S-11S.

2. Llewellyn CD, Johnson NW, Warnakulasuriya KA. Risk factors for squamous cell carcinoma of the oral cavity in young people: A comprehensive literature review. Oral Oncology $2001 \mathrm{Jul}$; 37(5):401-18.

3. Chen IH, Chang JT, Liao CT, et al. Prognostic significance of EGFR and Her-2 in oral cavity cancer in betel quid prevalent area cancer prognosis. Br J Cancer 2003 Aug 18;89(4):681-86.

4. Tanaka $T$, Ishigamori R. Understanding carcinogenesis for fighting oral cancer. J Oncol 2011, Article ID 603740, 10 pages.

5. Petersen PE. Oral cancer prevention and control: The approach of the World Health Organization. Oral Oncol 2009;45(4-5): 454-60.

6. Subapriya, Rajamanickam, Thangavelu, et al. Assessment of risk factors for oral squamous cell carcinoma in Chidambaram, Southern India: A case-control study. Europ J Can Prev 2007 June;16(3):251-56

7. Waranakulasuriya S, Sutherland G, Scully. Tabacco, oral cancer and treatment of dependence. Oral Oncol 2005;41:244-60.

8. Velly AM, Franco EL, Schlecht N, et al. Relationship between dental factors and risk of upper aerodigestive tract cancer. Oral Oncol 1998;34(4):284-91.

9. Reibel J. Prognosis of oral premalignant lesions: Significance of clinical, histopathological and molecular biological characteristics. Crit Rev Oral Biol Med 2003;14:47.

10. Lee JJ, et al. Predicting cancer development in oral leukoplakia: Ten years of translational research. Clin Can Res 2000;6:1702-10.

11. Lodi G, Sardella A, Bez C. Interventions for treating oral leukoplakia (Review). Cochrane Database Syst Rev 2006 Oct 18;4:CD001829.

12. Liu SC. Marker of cell proliferation in normal epithelia and dysplastic leukoplakias of the oral cavity. Cancer Epidemiol Biomarkers Prev 1998;7:597-603.

13. Scholzen T, Gerdes J. The Ki-67 protein from the known and the unknown. J Cell Physiol 2000;182:311-22.

14. Jonat W, Arnold N. Is the Ki-67 labelling index ready for clinical use? Annals Oncol 2011;22:500-02. 
15. Schlfiter C, Duchrow M, Wohlenberg C, et al. The cell proliferation-associated antigen of antibody Ki-67: A very large, ubiquitous nuclear protein with numerous repeated elements, representing a new kind of cell cycle-maintaining proteins. J Cell Biol 1993;123(3):513-22.

16. Zidar N, Gale N, Cor A, Kambic V. Expression of Ki67 antigen and proliferative cell nuclear antigen in benign and malignant epithelial lesions of the larynx. J laryngol Otol 1996;110:440-45.

17. Macluskey M. The association between epithelial proliferation and disease progression in the oral mucosa. Oral Oncol 1999;35: 409-14.

18. Slootweg PJ, Koole R, Hardicjk GJ. The presence of p53 in relation to Ki67 as a cellular proliferation marker in head and neck squamous cell carcinoma and adjacent dysplastic mucosa. Oral Oncol Europ J Can 1994;30(B);138-414.

19. Todd R, Hinds PW, Munger K, et al. Cell cycle dysregulation in oral cancer. Crit Rev Oral Biol Med 2002;13:151-61.

20. Carreras R, Alameda F, Mancebo G. A study of Ki-67, c-erbB2 and cyclin D-1 expression in CIN-I, CIN-III and squamous cell carcinoma of the cervix. Histol Histopathol 2007;22:587-92.

21. Stanton KJ, Sidner RA, Miller GA. Analysis of Ki-67 antigen expression, DNA proliferative fraction, and survival in resected cancer of the pancreas. Am J Surg 2003;186(5):486-92.

22. Sym S, Hong J, Cho E, et al. Prognostic impact of immunohistochemical expression of $\mathrm{Ki}-67$ in patients with advanced gastric cancer who underwent curative resection. J Clin Oncol 2011; (Supl 4):29.

23. Margulis V, Shariat SF, Ashfaq R. Ki-67 is an independent predictor of bladder cancer outcome in patients treated with radical cystectomy for organ-confined disease. Clin Can Res 2006 Dec 15;12(24):7369-73.

\section{ABOUT THE AUTHORS}

\section{Veena Maheshwari (Corresponding Author)}

Professor, Department of Pathology, JN Medical College, Aligarh Uttar Pradesh, India, e-mail: vegovil@gmail.com

\section{SC Sharma}

Professor, Department of ENT, JN Medical College, Aligarh, Uttar Pradesh, India

\section{Varsha Narula}

Junior Resident, Department of Pathology, JN Medical College Aligarh, Uttar Pradesh, India

\section{Sachin Verma}

Junior Resident, Department of ENT, JN Medical College, Aligarh Uttar Pradesh, India

\section{Anshu Jain}

Assistant Professor, Department of Pathology, JN Medical College Aligarh, Uttar Pradesh, India

\section{Kiran Alam}

Associate Professor, Department of Pathology, JN Medical College Aligarh, Uttar Pradesh, India 